\title{
Desigualdades socioeconómicas y distribución inequitativa de los riesgos ambientales en las comunas de Peñalolén y San Pedro de la Paz. Una perspectiva de justicia ambiental ${ }^{1}$
}

\author{
Alexis Vásquez ${ }^{2}$ y Marcela Salgado ${ }^{2}$
}

\begin{abstract}
RESUMEN
El desarrollo de las ciudades latinoamericanas se ha caracterizado por un proceso inadecuado de apropiación de la naturaleza, que puede ser observado en la fragmentación del espacio urbano y en la distribución desproporcionada de amenazas y amenidades ambientales entre distintos grupos socioeconómicos. Para analizar y evaluar la interrelación espacial existente entre la distribución de amenazas ambientales y los niveles socioeconómicos de la población y, luego, analizar la distribución de dichas amenazas y su relación con mecanismos que actúan sobre la vulnerabilidad física de los territorios ocupados por los diferentes grupos socioeconómicos, se han escogido las comunas de San Pedro de la Paz y Peñalolén. En el caso de San Pedro de la Paz se constató una mayor exposición de los sectores pobres a las amenazas ambientales, lo que no ocurre en el caso de Peñalolén donde la diferencia en los niveles de ingreso actúa como un factor determinante en la disminución de la vulnerabilidad física.
\end{abstract}

Palabras clave: Amenazas ambientales, desigualdades socioeconómicas, justicia ambiental.

\begin{abstract}
The development of Latin American cities has been characterized by an inadequate process of nature appropriation, that could be observed in the urban space fragmentation and in the disproportionate distribution of environmental threaten and amenities among different socioeconomic groups of inhabitants. To analyze and assess spatial relationship between environmental risks and socioeconomic groups, two urban communes, Peñalolen and San Pedro de la Paz, were chosen. Further, the distribution of such threats and their relations with mechanisms that are acting over the physical vulnerability of socioeconomic groups were taken into consideration. In the case of San Pedro de la Paz, it is possible to observe a large exposition of the poorest people to environmental threats. In the case of Peñalolen, income level differences are acting as a determinant factor in the capacity to reduce physical vulnerability.
\end{abstract}

Key words: Environmental threats, socioeconomic disparities, environmental justice.

Proyecto FONDECYT No 1050423. Artículo recibido el 13 de marzo de 2008 y aceptado el 22 de abril de 2009 .
2 Laboratorio Medio Ambiente y Territorio, Universidad de Chile (Chile). E-mail: alexvasq@uchile.cl; salgado.marcela@gmail.com 
El modelo predominante en las ciudades latinoamericanas es el de ciudad fragmenta$d a$, caracterizada por paisajes urbanos cada vez más complejos y heterogéneos, donde la distancia física entre los espacios ocupados por los distintos grupos socioeconómicos -como representación de la distancia social- se ha reducido en los últimos años, adoptando nuevos símbolos y significados. Se advierte en la región un cambio en la escala de segregación socioespacial, siendo cada vez más frecuente encontrar barrios de todos los niveles socioeconómicos en una misma comuna (Hidalgo, 2004; Borsdorf et al., 2006). Estas nuevas formas y magnitudes de segregación social y espacial tienen su mayor expresión en estas escalas locales de análisis (Borsdorf et al., 2006).

Por otra parte, el desarrollo de las ciudades en Latinoamérica no ha considerado adecuadamente la degradación ambiental y ha construido infraestructuras sin considerar criterios suficientes de seguridad contra las amenazas, riesgos e incertidumbres naturales, que, debido a procesos como el cambio climático, generan escenarios completamente diferentes a los actuales. Ha existido un proceso inadecuado de apropiación de la naturaleza, una alta vulnerabilidad de la población pobre y gran inequidad en la distribución del ingreso (Lavell, 1999). Por ello, no parece extraño que un $90 \%$ de las víctimas de desastres naturales viva en países en desarrollo, bajo condiciones de alto riesgo (Vargas, 2002).

Los mecanismos del mercado, aceptados y aplicados sin restricciones en países como Chile, estarían acentuando una apropiación, comodificación y privatización cada vez más exclusiva de los espacios más valorizados dentro de las ciudades (Romero y Vásquez, 2005b). En este sentido, se asume que la distribución de la población en los espacios urbanos -y por lo tanto su accesibilidad a medios ambientes de calidad y mayor seguridad ante los riesgos- se encuentra cada vez más fuertemente condicionada por el nivel de ingreso de cada grupo social. Por lo tanto, grupos de menores ingresos terminan accediendo a las zonas ambientalmente más degradadas, más contaminadas y que presentan mayores amenazas de la ciudad $y$, por tanto, se configuran asentamientos que concentran mayores grados de vulnerabilidad frente a amenazas ambientales o peores efectos sobre la salud de las personas y de los ecosistemas (Vásquez et al., 2008).

Existen evidencias de que en diversas ciudades los efectos medioambientales negativos causados, por ejemplo, por los depósitos de residuos tóxicos no se distribuyen de manera homogénea en la población, sino más bien se aprecia una sobrecarga de estos problemas en sectores habitados por comunidades de bajos ingresos o pertenecientes a alguna minoría étnica (EPA, 2002; Walker and Bulkeley, 2006; Krieg and Faber, 2004; Fisher et al., 2006). Adicionalmente, existen antecedentes para pensar que al igual que los efectos ambientales negativos, las funciones ambientales favorables, como las otorgadas por la vegetación, también se distribuyen de manera asimétrica en la población (Pedlowski et al., 2002; Iverson \& Cook, 2000; De la Maza et al., 2002; Escobedo et al., 2006), siendo más escasas en los sectores pobres, que al mismo tiempo son los más deteriorados por impactos ambientales acumulativos. Entre los servicios ambientales prestados por la vegetación en las ciudades, se encuentra regular el clima, mejorar la calidad del aire, proporcionar hábitat a especies biológicas, elevar la calidad estética y favorecer la infiltración de las aguas lluvias en el suelo, disminuyendo los volúmenes de escurrimiento superficial y las inundaciones (Pedlowski et al., 2002). Todas estas funciones han demostrado ser eficaces en la disminución de la vulnerabilidad de los habitantes de las ciudades frente a amenazas ambientales tales como calor extremo, inundación y anegamiento.

La ocurrencia de desastres y la exposición a los riesgos ambientales parecen no ser resultado de una naturaleza castigadora sino más bien de la configuración social de las vulnerabilidades. Tal como señala Hipple (2007), el riesgo de que ocurra un desastre se convierte en un concepto clave al momento de abordar esta problemática, de tal manera que la reducción de su incidencia requiere de estudios sobre la configuración social del riesgo. En este sentido, el riesgo debe ser entendido en función de sus dos componentes centrales: i) amenaza o peligro potencial, y ii) vulnerabilidad frente a di- 
chas amenazas (Cardona, 2001; Hipple, 2007). En otras palabras, para que exista riesgo debe haber una condición de amenaza frente a la cual se está expuesto y se es sensible; no se puede ser vulnerable si no hay amenaza y esta no es tal si no hay un sistema frágil ante ella (Cardona, 2001). Al incorporar la noción de vulnerabilidad como componente del riesgo, finalmente se confiere responsabilidad a la sociedad frente a la ocurrencia de desastres. Por lo tanto, se habla de desastres socionaturales al referirse a eventos que son autoproducidos por el sistema social.

La vulnerabilidad de los sistemas sociales hace referencia a la escasa capacidad de respuesta individual o grupal de la sociedad ante riesgos y contingencias y, también, a la predisposición a la caída en el nivel de bienestar, derivada de una configuración de atributos negativos para lograr retornos materiales y simbólicos. Por extensión, se puede afirmar que es también una predisposición negativa para la superación de condiciones adversas (Filgueira y Peri, 2004).

La vulnerabilidad física de un asentamiento humano puede ser entendida como la susceptibilidad que tiene a ser afectado por un desastre debido, por una parte, al estar en el área de influencia de las amenazas naturales $y$, por otra, por su falta de resistencia física (infraestructura, equipamiento, materialidad, entre otros) ante las mismas (Cardona, 2001).

En Chile casi nueve de cada diez personas vive en ciudades (INE, 2002). En los últimos años, las Áreas Metropolitanas de Santiago y Concepción han incrementado su tamaño y la velocidad de su expansión física (Romero y Vásquez, 2007a; Romero y Vásquez, 2005a; Romero y Vásquez, 2005b) pese a que sus tasas de crecimiento poblacional se desaceleran. La aplicación sostenida de las premisas neoliberales a la gestión de los territorios urbanos en Chile presenta claros signos de insustentabilidad al cabo de más de treinta años, manifestada en el crecimiento ilimitado de las áreas urbanizadas, el fortalecimiento de la segregación socioambiental y el aumento de las diferencias de calidad ambiental en las áreas y paisajes ocupados por los estratos ricos y pobres de la población urbana. Estudios recientes han demostrado cómo en la ciudad de Santiago los espacios ocupados por el grupo socioeconómico (GSE) D son los más expuestos a calor extremo, elevadas concentraciones de Material Particulado (MP10) en la atmósfera y carencia de espacios vegetados (Romero \& Vásquez, 2007a, 2007b).

\section{Área de Estudio}

Las comunas de San Pedro de la Paz y Peñalolén son representativas del nuevo modelo de urbe latinoamericana donde la alta complejidad y heterogeneidad socioespacial a escala intracomunal se convierten en rasgos característicos, la emergencia y desarrollo de este tipo de ciudad fragmentada platea la reproducción creciente de este patrón en las demás comunas de las áreas metropolitanas chilenas.

\section{Comuna de San Pedro de la Paz}

La comuna de San Pedro de la Paz pertenece al Gran Concepción, Región del Biobío (Chile). Se localiza al sur poniente del río Biobío, a $36^{\circ} 50^{\prime}$ de latitud Sur y $73^{\circ} 05^{\prime}$ de longitud Oeste, contando con una superficie de $112,5 \mathrm{~km}^{2}$ entre los que se encuentran 14 $\mathrm{km}$ de costa, $22 \mathrm{~km}$ de ribera en el río y dos lagunas naturales (Figura $N^{\circ} 1$ ).

De acuerdo a los datos del último Censo de Población y Vivienda del año 2002, San Pedro de la Paz es una de las comunas más pobladas de las 52 que forman parte de la Región del Biobío, concentrando una población total de 80.447 habitantes, de los cuales un 99,6\% habita en áreas urbanas y un $0,4 \%$ en áreas rurales. Es importante destacar que en los últimos años esta comuna ha sido receptora de importantes contingentes de población provenientes de otras comunas del Área Metropolitana de Concepción, de la Región del Biobío y de otras zonas geográficas del país. Al respecto, los datos censales indican que un $27,7 \%$ de la población residía en San Pedro de la Paz hace 5 años y un $70,7 \%$ lo hacía en otra comuna de la región o del país. Como consecuencia del aumento de población que ha experimentado la comuna, diversos proyectos inmobiliarios han comenzado a ocupar sectores de cerros 
próximos a las lagunas Grande y Chica de San Pedro, humedales y dunas costeras para actividades residenciales y de equipamiento.

\section{Comuna de Peñalolén}

La comuna de Peñalolén, una de las diez comunas de mayor extensión territorial de la provincia de Santiago, tiene una superficie de $54,9 \mathrm{~km}^{2}$, representando un $0,3 \%$ de la $\mathrm{Re}$ gión Metropolitana de Santiago. De la extensión total de la comuna, el $58,6 \%$ es considerado como área urbana.

Desde el punto de vista topográfico se distinguen tres zonas: i) el valle bajo del canal San Carlos, con una altura promedio de 560 m.s.n.m.; ii) la zona que va desde el canal San Carlos hasta la falla de San Ramón, a 900 m.s.n.m., donde se encuentra Peñalolén Alto y la viña Cousiño Macul, y iii) la zona que va desde la falla hasta la cumbre del cerro San Ramón, sobre los 3.000 m.s.n.m., donde se encuentran las quebradas de Peñalolén y Macul (Figura № 1 ).
En la comuna de Peñalolén, el 2002 habitaban 216.060 personas, ubicándose dentro de las doce más pobladas del país y en la sexta posición en relación a la Región Metropolitana de Santiago (INE, 2002). De acuerdo a las proyecciones del INE para el año 2007 la población aumentaría a alrededor de 240.032 habitantes.

\section{Objetivo}

En términos generales, este estudio pretende aportar antecedentes a la discusión científica y social respecto a los problemas de distribución social de los "males" y "bienes" ambientales, a través de la evaluación de la interrelación espacial existente entre la distribución de las amenazas ambientales y los niveles socioeconómicos de la población, en las comunas de Peñalolén y San Pedro de la Paz. Adicionalmente, se busca analizar la distribución de las amenazas ambientales y su relación con algunos de los mecanismos que actúan sobre la vulnerabili-
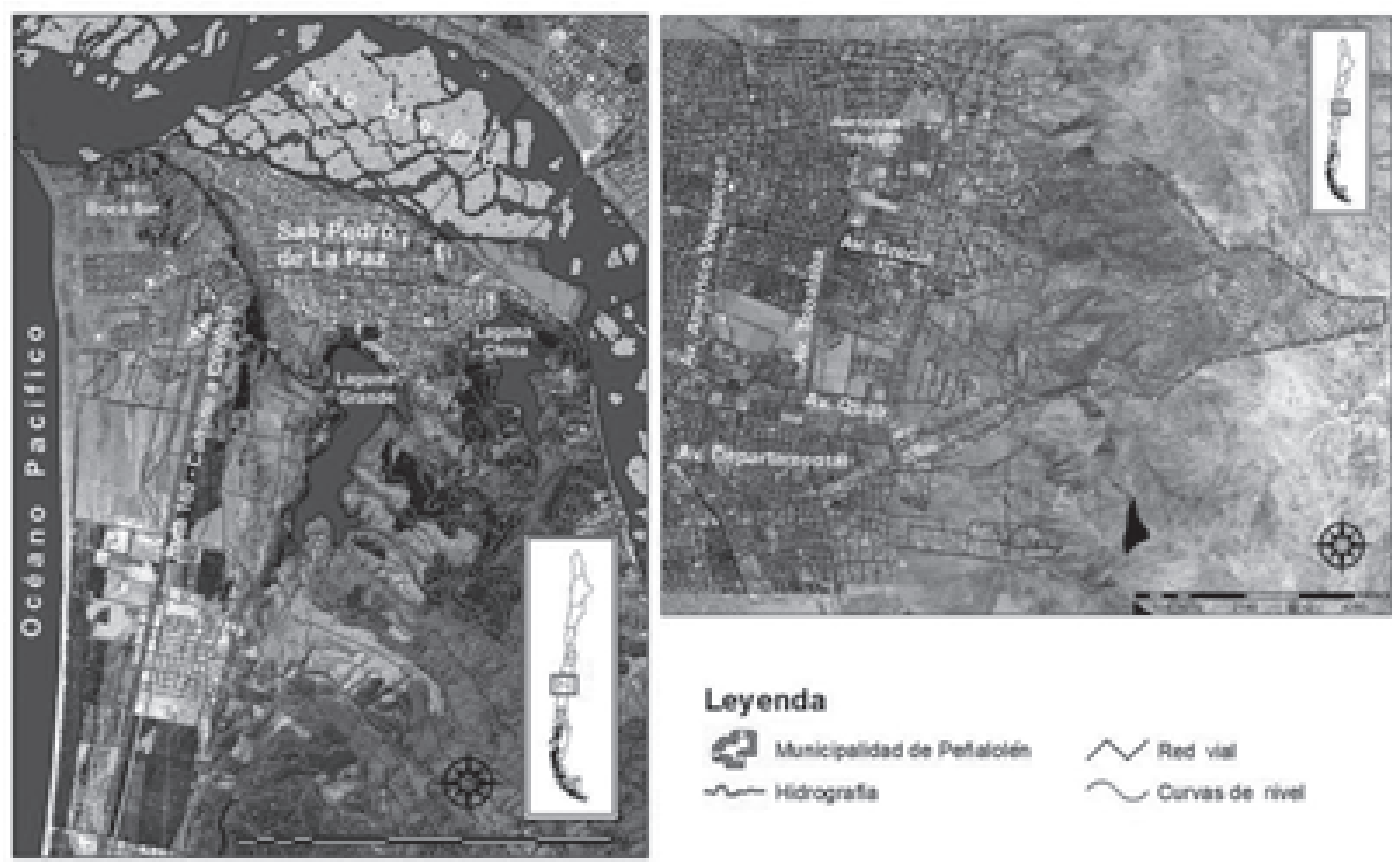

Fuente: Elaboración propia.

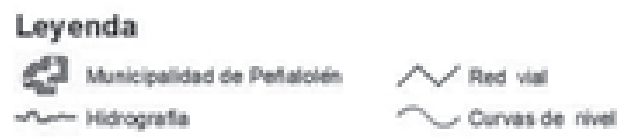


dad física de los territorios ocupados por diferentes grupos socioeconómicos.

\section{Metodología}

Para obtener el nivel socioeconómico de la población a nivel de manzana, se procesó información del Censo de Población y Vivienda 2002 del Instituto Nacional de Estadística, a través del sistema computacional REDATAM+ G4. La clasificación socioeconómica empleada considera el nivel de escolaridad del jefe(a) de hogar y la cantidad de bienes que posee cada hogar (Adimark, 2002).

Para determinar las amenazas ambientales se emplearon los estudios de riesgos de los Planes Reguladores de ambas comunas. Las coberturas utilizadas fueron las correspondientes amenazas de inundación, anegamiento y remoción en masa. En San Pedro de la Paz también se consideraron las informaciones de amenaza por inundación mareal, deflación eólica, descarga de aguas contaminadas y vertederos de residuos sólidos no autorizados.

El estudio de la distribución de la vegetación pública y privada se realizó a partir del análisis y procesamiento de imágenes satelitales Landsat TM del año 2007 y del año 2005 para Peñalolén y San Pedro de la Paz, respectivamente. La clasificación digital de las imágenes fue de carácter supervisada (donde las firmas espectrales de los objetos, obtenidas desde el satélite, son identificadas con tipos de usos y coberturas del suelo mediante observaciones de terreno) y a una escala de cuadrículas de 30×30 m.

Para relacionar espacialmente la distribución de las amenazas ambientales con las superficies ocupadas por los diferentes grupos socioeconómicos que se encuentran expuestos a los efectos ambientales negativos considerados, se emplearon procedimientos de geoprocesamiento y modelos de correlaciones lineales. Los geoprocesos permitieron el ajuste, superposición e intersección espacial de las imágenes analizadas. Para evaluar las correlaciones lineales entre cobertura vegetal (pública y privada de manera conjunta) y los grupos socioeconómicos (GSE) se utilizó el coeficiente de correlación de Pearson y se construyeron diagramas de dispersión. Además, se calculó el coeficiente de determinación, que permite conocer la medida en que los cambios de la variable independiente logran explicar los de la variable dependiente.

Finalmente se realizó una descripción de los mecanismos que actúan sobre la vulnerabilidad física de los GSE, considerando sobre todo la distribución de las áreas verdes públicas y las obras civiles para el control de las inundaciones en la comuna de Peñalolén.

\section{Resultados}

\section{Distribución de las amenazas \\ ambientales según grupos \\ socioeconómicos}

La Figura № 2 muestra la distribución espacial de las amenazas en ambas comunas. Se observa que gran parte de los territorios se encuentra bajo alguna amenaza, debido principalmente a la localización en sectores donde los procesos geofísicos se manifiestan con gran fuerza. En el caso de Peñalolén se trata básicamente de los efectos causados por los flujos de materia y energía que se desplazan aguas abajo desde la cordillera de los Andes, dirigiéndose hacia la Depresión Intermedia o valle de Santiago, principalmente a través de un gran número de quebradas que existen en el piedemonte andino.

San Pedro, en cambio, está dominada por los procesos oceanográficos de la costa del océano Pacífico y la dinámica hidrológica del sistema laguna Grande, laguna Chica y humedal Los Batros, lo que se manifiesta en una mayor diversidad de amenazas ambientales. Adicionalmente, se presentan puntos de descarga de aguas contaminadas, principalmente emisarios sanitarios, que se localizan en las zonas ribereñas del río Biobío, del océano Pacífico y en las lagunas de agua dulce. Los basurales ilegales presentan una distribución lineal siguiendo la costa marina.

En la Figura $\mathrm{N}^{\circ} 3$ se observa la distribución de los grupos socioeconómicos. En el caso de Peñalolén se aprecia una clara dife- 
Figura $\mathrm{N}^{\circ} 2$

Distribución de amenazas en las comunas de San Pedro de la Paz y Peñalolén
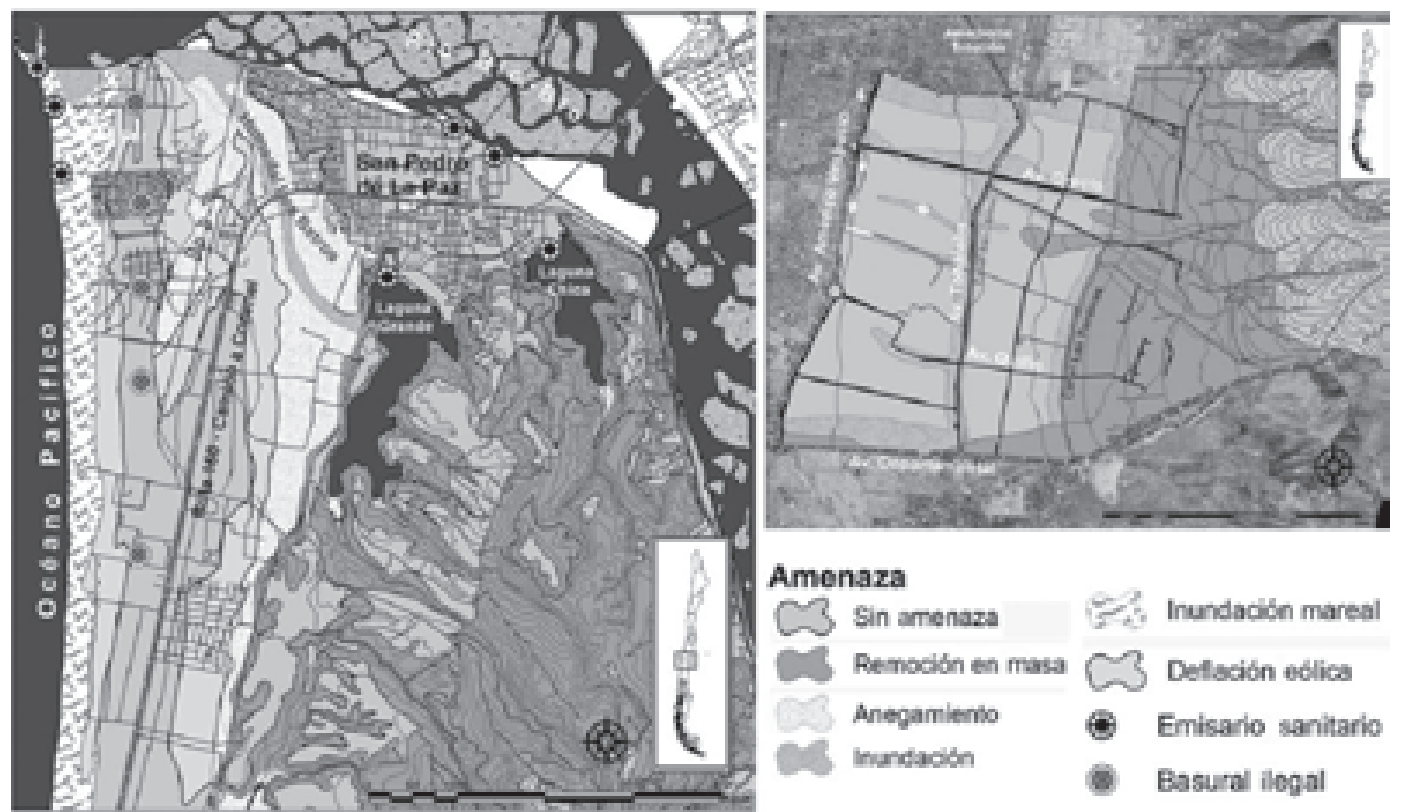

\section{Amenaza}

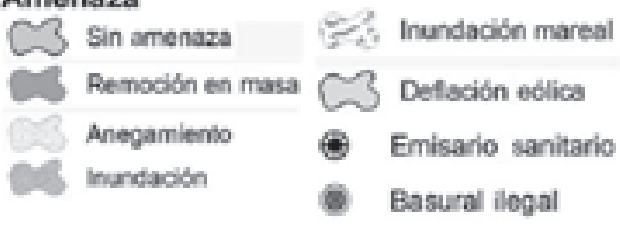

Fuente: Elaboración propia en base a estudio de riesgo del Plan Regulador Comunal y datos del Centro EULA de Ciencias Ambientales de la Universidad de Concepción.

Figura $\mathrm{N}^{\circ} 3$

Distribución de GSE en las comunas de San Pedro de la Paz y Peñalolén

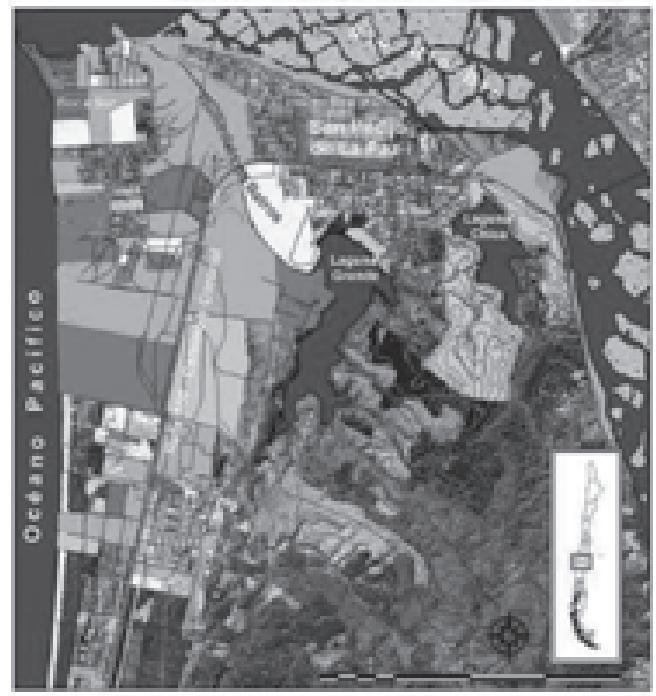

Fuente: Elaboración propia.

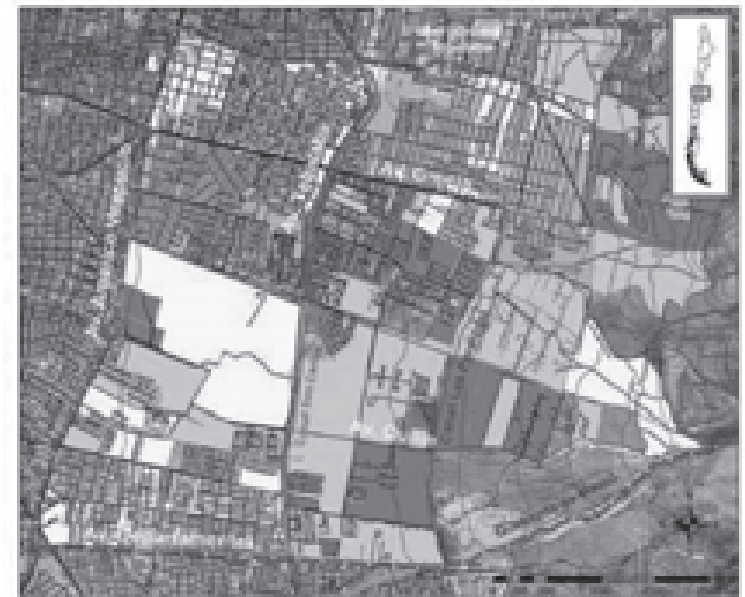

\section{Leyenda}

Grupo socioeconómico

C3

ADC1

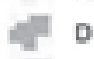

달. $\mathrm{C2}$
AI $\mathrm{D}$

ate 
rencia entre los sectores más antiguos y pertenecientes predominantemente a niveles socioeconómicos medios bajos (D), localizados al poniente de Av. Tobalaba y al norte de Av. Grecia, y los asentamientos de altos ingresos $(A B C 1)$, generalmente en forma de condominios cerrados, que recientemente han ido trepando por el piedemonte andino, alcanzando los terrenos más altos y escarpados de la comuna. Es interesante notar como Av. Grecia al oriente de Av. Tobalaba actúa como una línea divisoria entre espacios ocupados por GSE muy diferentes.

En San Pedro de la Paz, la llegada de habitantes de altos ingresos (ABC1) también ha sido reciente y ha estado focalizada en las áreas cercanas a las lagunas, donde se concentran amenidades ambientales asociadas a ellas. Asimismo, la zona costera, que en sus inicios fue casi exclusivamente ocupada por GSE bajos (D y E), ha atraído nuevos proyectos inmobiliarios destinados a los sectores más adinerados. En el caso de esta comuna, se relaciona la localización de basurales ilegales con los niveles socioeconómicos de la población, pues se aprecia que, si bien estos se distribuyen de manera lineal a través de seis kilómetros (Figura $N^{0} 4$ ), nunca se localizan en sectores habitados por los GSE más ricos. Por el contrario, la ubicación de los depósitos de residuos tiene una alta correspondencia espacial con la distribución de los GSE de menores ingresos (D y E).

Figura $\mathrm{N}^{\circ} 4$

Distribución de los basurales ilegales en relación con los niveles socioeconómicos en la comuna de San Pedro de la Paz

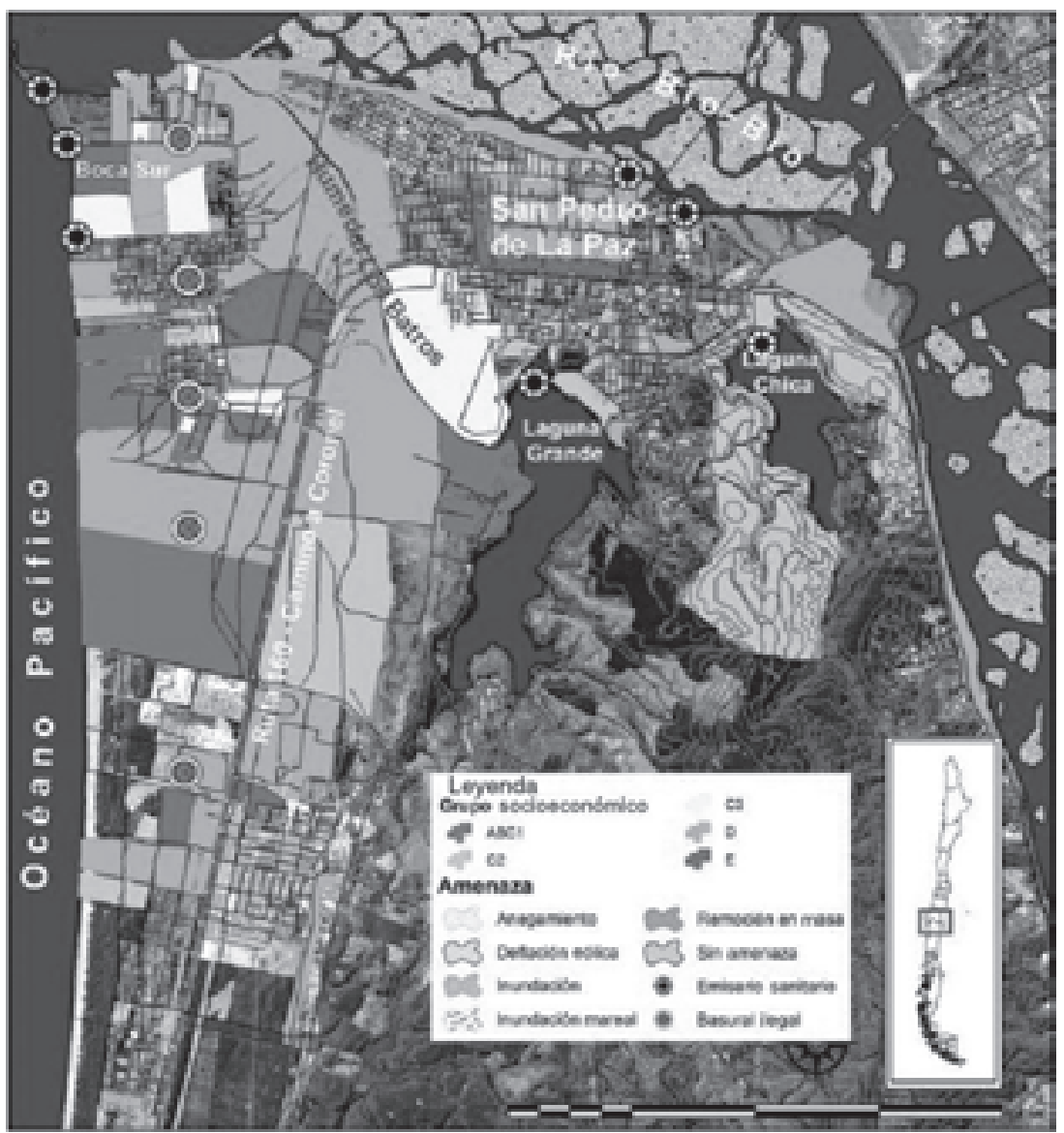

Fuente: Elaboración propia en base a datos del centro EULA. 
La Figura $N^{\circ} 5$ muestra las superficies ocupadas por los distintos grupos socioeconómicos, $100 \mathrm{~m}$ en torno a los vertederos clandestinos. El único GSE que en ninguno de los casos se localiza a menos de $100 \mathrm{~m}$ de los basurales es el $A B C 1$, incluso considerando que este grupo se encuentra muy bien distribuido en la zona costera. En términos generales, la superficie ocupada por los distintos GSE aumenta de manera sistemática en la medida que decaen los niveles de riqueza, a excepción del grupo socioeconómico $\mathrm{E}$, que si bien ocupa superficies mayores que los grupos $A B C 1, C 2$ y C3, se ubica muy por debajo del GSE D. Este último grupo ocupa un $86 \%$ de los sectores próximos a los vertederos, siendo el más afectado por sus efectos ambientales negativos tales como olores, vectores sanitarios y deterioro de la calidad paisajística. Los grupos C2 y C3 no alcanzan valores significativos.

En general, las descargas de aguas contaminadas ocurren en sectores deshabitados (Figura $N^{\circ} 4$ ), aunque se pueden identificar claramente dos puntos localizados en lagunas muy cercanas a los sectores residenciales del estrato $\mathrm{C} 2$, predominantes en esa área. Otros dos puntos de descarga de aguas contaminadas se ubican en la ribera del río Biobío y en la costa marina, en si- tios ocupados por sectores sociales D y E, respectivamente.

Es posible observar que los hogares de nivel socioeconómico más alto (ABC1) se ubican en un $47,7 \%$ en zonas exentas de amenazas (Figura $N^{\circ}$ 6). Sin embargo, un $20,3 \%$ y un $16 \%$ de la superficie ocupada por este GSE se encuentra bajo la amenaza de inundación mareal y deflación eólica, respectivamente, debido antes que todo a su reciente y desaprensiva localización costera (Figura $N^{\circ} 7$ ).

Aun cuando el $35,9 \%$ de la superficie ocupada por el grupo de nivel socioeconómico $D$ se localiza en sectores sin amenaza (Figura $\mathrm{N}^{\circ} 8$ ), el $48,1 \%$ de las áreas en que reside esta población de ingresos medios bajos está expuesta a amenazas de anegamiento y deflación eólica. En el caso de los anegamientos, afectan mayoritariamente a los habitantes de este grupo socioeconómico localizados en sectores cercanos o pertenecientes al humedal Los Batros.

Es interesante destacar que existe un alto porcentaje de la superficie ocupada por el nivel socioeconómico $A B C 1$ expuesto a remociones en masa, como consecuencia principalmente de la ocupación de sectores escarpados sobre los cerros en torno a ambas lagunas.

Figura $\mathrm{N}^{\circ} 5$

Superficies ocupadas por los diferentes GSE $100 \mathrm{~m}$ a la redonda en torno a vertederos ilegales

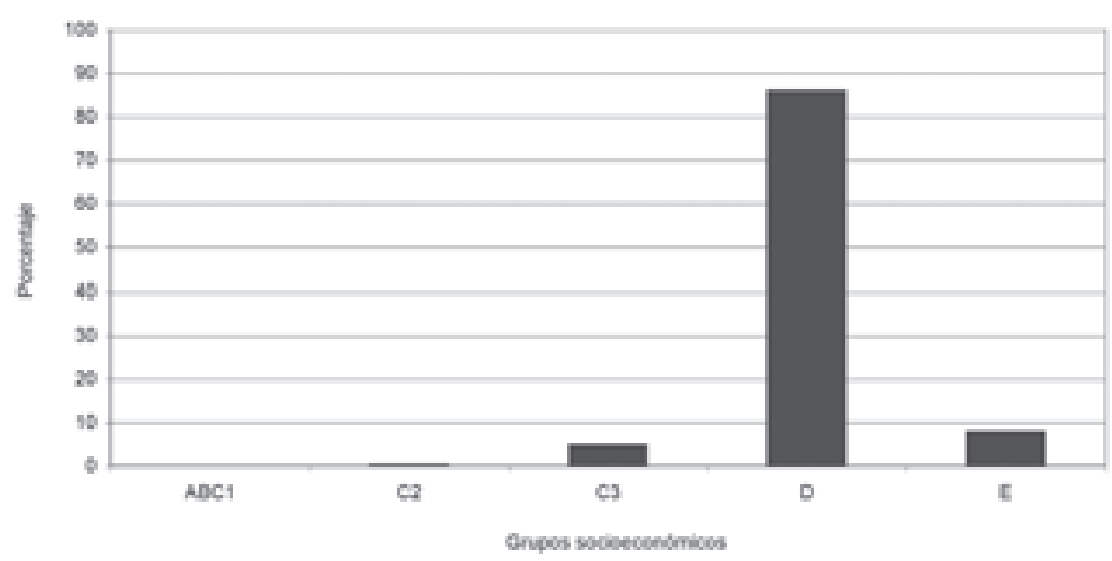

Fuente: Elaboración propia. 
Figura $\mathrm{N}^{\circ} 6$

Exposición de los espacios ocupados por niveles socioeconómicos $\mathrm{ABC} 1$ a amenazas de origen natural en la comuna de San Pedro de la Paz

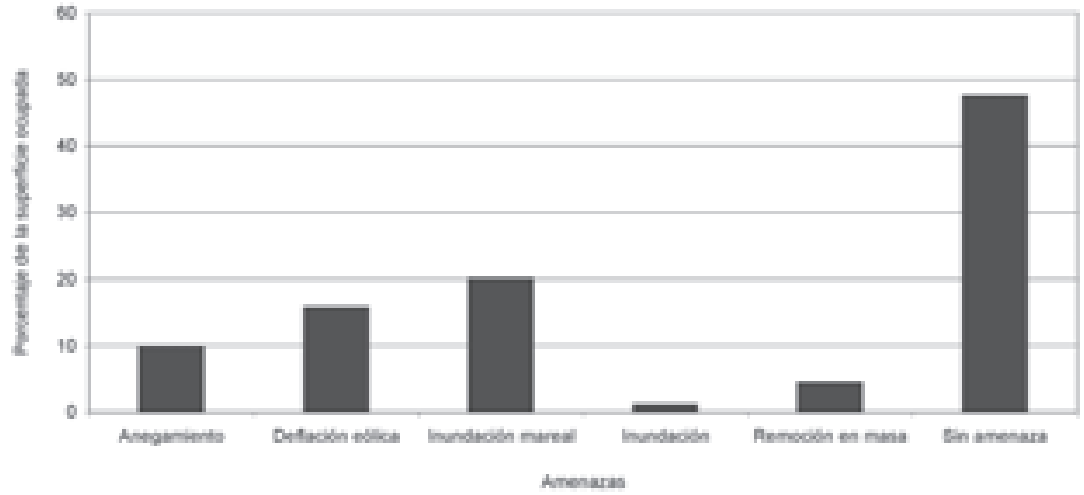

Fuente: Elaboración propia.

Figura $\mathrm{N}^{\circ} 7$

Distribución de las amenazas ambientales para los GSE ABC1 y D en la comuna de San Pedro de la Paz

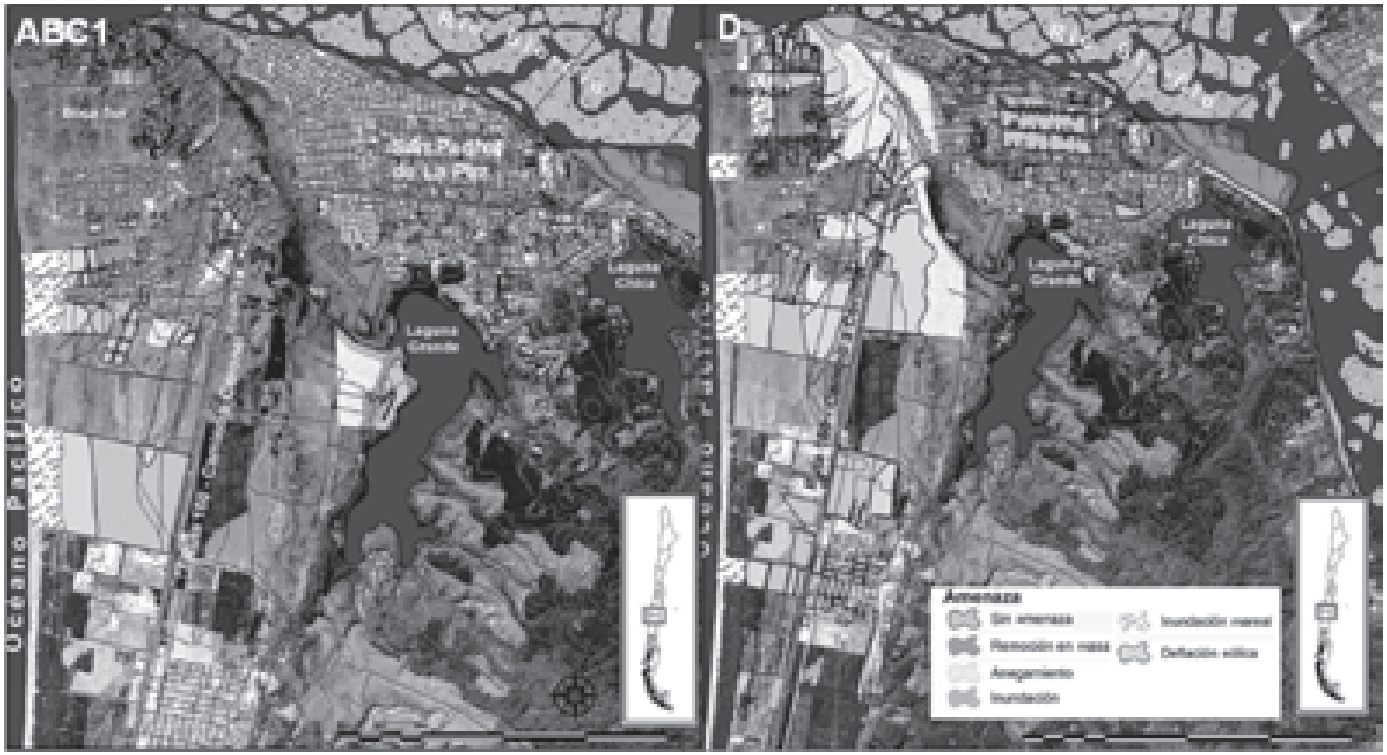

Fuente: Elaboración propia en base a estudio de riesgo del Plan Regulador Comunal.

En el caso de Peñalolén la situación es opuesta, ya que son los habitantes de altos ingresos $(A B C 1)$ los que viven en áreas mayormente expuestas a las amenazas de remoción en masa e inundación. La Figura $\mathrm{N}^{\circ}$
9 muestra que el $67,1 \%$ de la superficie habitada por hogares ABC1 se encuentra bajo algún tipo de amenaza, siendo más significativa la exposición a remociones en masa, con 53,9\% del total. Por el contrario, el 
$45,4 \%$ de los espacios ocupados por el nivel socioeconómico medio bajo (D) se ubican en sectores sin amenaza (Figura $N^{\circ} 10$ ).

La diferencia en los tipos y magnitudes de las amenazas a las que se ven expuestos estos dos GSE, se explica fundamentalmente por la posición que ocupan dentro de la cuenca que da origen a la comuna de Peñalolén. En la Figura $N^{0} 11$ se observa claramente que los sectores $A B C 1$ alcanzan una marcada exposición a remociones en masa de sedimentos debido a que se localizan en sectores inclinados e inestables de los conos de deyección y cercanos a múltiples quebradas. Estas áreas están dentro de las más demandadas para proyectos inmobiliarios debido a que presentan grandes amenidades ambientales tales como calidad escénica.

Por el contrario, la localización inicial de los sectores más pobres en la parte baja de la cuenca hace que estén expuestos sobre todo a inundaciones, producidas por la acumulación de volúmenes de agua provenientes desde la parte alta de las cuencas, conducidas inicialmente por sus cauces naturales (quebradas), que luego penetran en el sector urbano generalmente en forma de calles y avenidas pavimentadas.
También es importante la presencia de los canales de riego, como el canal San Carlos y el canal Las Perdices, que atraviesan la comuna. Ambos tienen una marcada incidencia en la ocurrencia de inundaciones. El canal Las Perdices afecta más directamente a los sectores $A B C 1$, mientras que el San Carlos atraviesa sectores con predominio de grupos de menores ingresos (Figura $N^{\circ} 11$ ).

\section{Descripción de los mecanismos que actúan sobre la vulnerabilidad física de los grupos socioeconómicos}

La diferencia en los niveles de ingreso para cada grupo puede ser entendida como un factor determinante en la configuración de vulnerabilidades. En este sentido, los hogares del estrato $\mathrm{D}$ no cuentan con la misma capacidad de acceso a ciertos servicios y bienes que les permitan reducir su vulnerabilidad frente a las amenazas a los cuales se ven expuestos.

Por ejemplo, la distribución de la vegetación en la comuna de Peñalolén está altamente asociada con los niveles socioeconómicos de la población. Si se considera como variable independiente el porcentaje de población ABC1 de cada manzana censal y

\section{Figura $\mathrm{N}^{\circ} 8$}

Exposición de los espacios ocupados por niveles socioeconómicos

$\mathrm{D}$ a amenazas de origen natural en San Pedro de la Paz

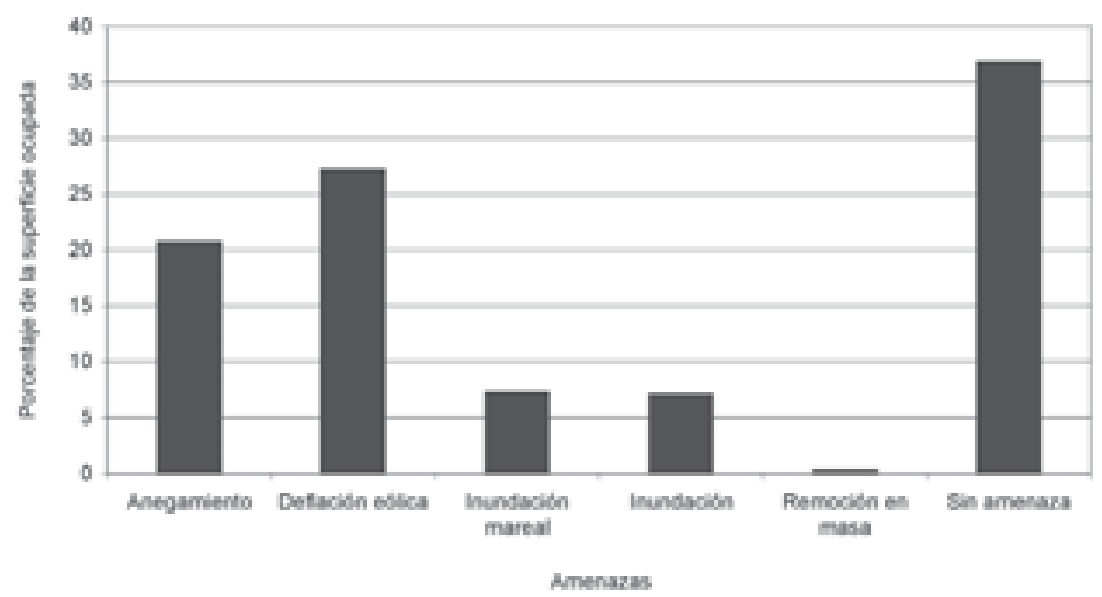

Fuente: Elaboración propia. 
como variable dependiente la cobertura vegetal de la misma manzana, la variación de esta última puede ser explicada en un $40 \%$ por los valores de la primera: mientras mayor sea el porcentaje de población $\mathrm{ABC} 1$ que vive en una manzana, la cobertura vegetal también será mayor (Figura № 12).
Por el contrario, la relación entre porcentaje de población del GSE D y la cobertura vegetal por manzana es inversa. Por lo tanto, mientras mayor sea el porcentaje de población de este nivel socioeconómico en una manzana, menor será la cobertura vegetal (Figura $N^{\circ} 13$ ).

Figura $\mathrm{N}^{\circ} 9$

Exposición de los espacios ocupados por nivel socioeconómico ABC1 a amenazas ambientales de origen natural en la comuna de Peñalolén

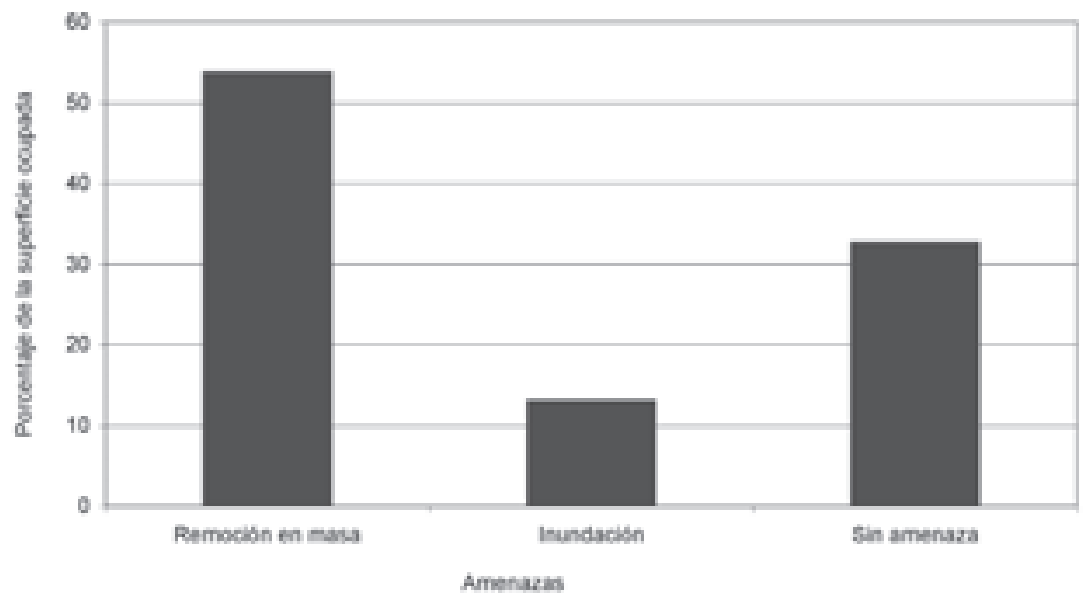

Fuente: Elaboración propia.

Figura $\mathrm{N}^{\circ} 10$

Exposición de los espacios ocupados por nivel socioeconómico D a amenazas de origen natural en la comuna de Peñalolén

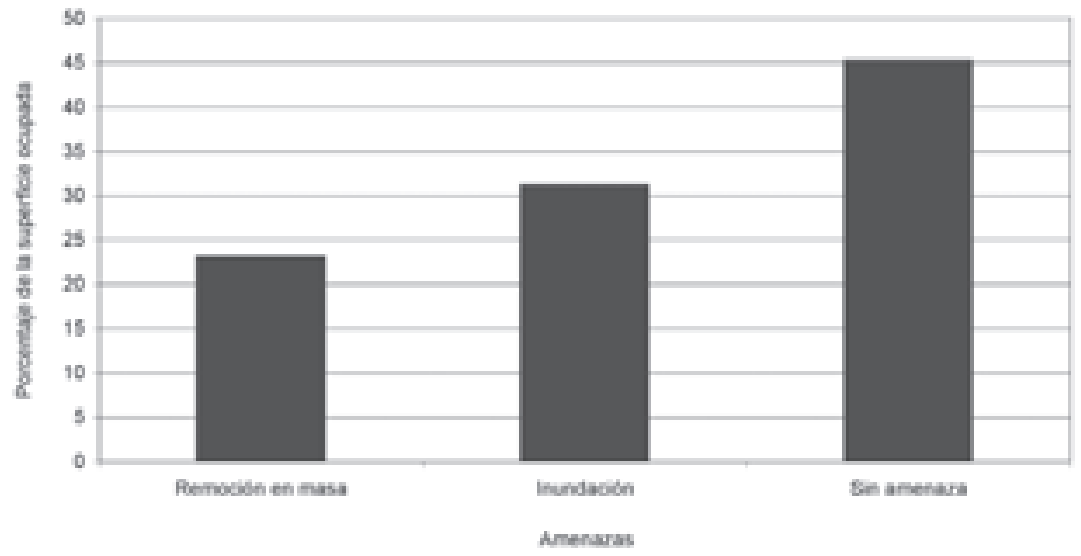

Fuente: Elaboración propia. 
Figura $N^{\circ} 11$

Distribución de las amenazas ambientales para los GSE ABC1 y D en la comuna de Peñalolén
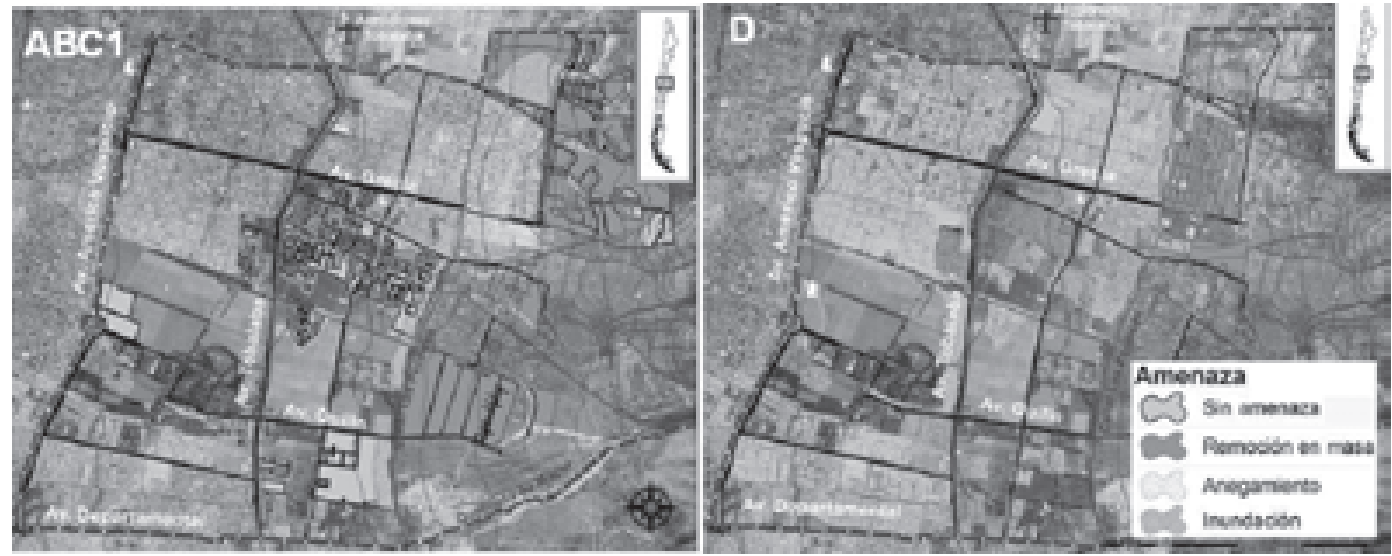

Fuente: Elaboración propia en base a estudio de riesgo del Plan Regulador Comunal.

Figura $\mathrm{N}^{\circ} 12$

Relación entre el porcentaje de población $\mathrm{ABC} 1$ y cobertura vegetal en la comuna de Peñalolén

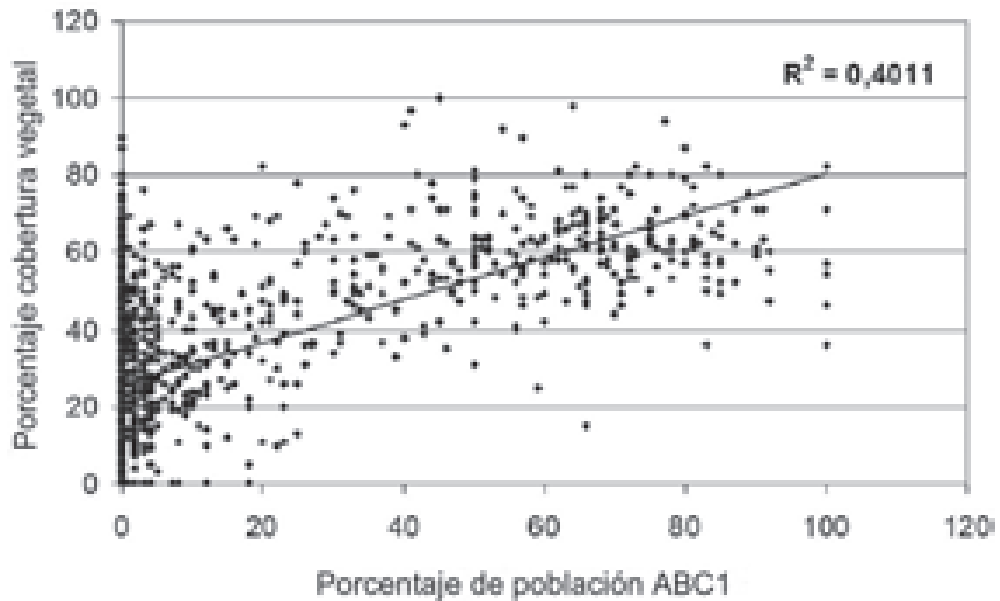

Fuente: Elaboración propia.

En el caso de la comuna San Pedro de la Paz estos tipos de relaciones no son estadísticamente significativas. Las correlaciones entre porcentaje de población de los diferentes GSE por manzana y la cobertura vegetal de las mismas fueron muy cercanas a cero. Romero et al. $(2001,2003)$ han atribuido esta situación a las condiciones geo- gráficas predominantes en las ciudades del sur de Chile, donde el clima favorece la aparición espontánea de vegetación y su mantención a lo largo del año, contrario a lo que ocurre en ciudades del centro y norte de Chile, donde es necesario invertir grandes sumas de dinero en riego y manejo de jardines y parques. 
Otro aspecto importante es el que se relaciona con la capacidad de ciertos grupos socioeconómicos de desplegar mecanismos que les permiten disminuir en cierto grado su vulnerabilidad frente a las amenazas naturales. Por ejemplo, en Peñalolén, un $67,1 \%$ de la superficie habitada por hogares $A B C 1$ se encuentra bajo algún tipo de amenaza, principalmente remoción en masa. Sin embargo, estos grupos cuentan con un nivel de ingreso tal que les permite acceder a proyectos residenciales privados, condominios cerrados que están dotados de ductos e infraestructuras de conducción de las aguas y los sedimentos fuera de sus límites (Figura $N^{0}$ 14). Es decir, el aumento en las tasas de impermeabilización y, consecuentemente, de los flujos de escorrentía de aguas lluvias y sedimentos, causado por la construcción de áreas residenciales en sitios del piedemonte andino, es transformado en externalidad negativa y trasladada aguas abajo, donde se localizan las áreas residenciales de sectores más vulnerables, originando un efecto desproporcionadamente alto. En este tipo de situaciones, cuando el mercado, las políticas, planes y las acciones gubernamentales derivan en situaciones de desigualdad, es cuando se está en presencia de una injusticia ambiental.
Por el contrario, si bien los hogares del grupo socioeconómico D tienden a ocupar espacios sin amenazas naturales, tienen mayores limitaciones de recursos al momento de protegerse, reaccionar y recuperarse en caso que se desencadene un desastre, especialmente si el aumento de los flujos es causado por la acumulación artificial de agua y sedimentos sobre calles que son utilizadas como continuación de los cauces fluviales que provienen de las áreas más altas de las cuencas urbanizadas.

En definitiva, distintos grupos socioeconómicos pueden presentar un nivel de exposición relativamente semejante frente a las amenazas naturales, como las inundaciones o remociones en masa de sedimentos, pero la diferencia en su comportamiento radica en que aquellos que tienen mayor capacidad de acceso a bienes y servicios pueden disponer individual o socialmente de infraestructuras apropiadas para prevenir y protegerse. Por otro lado, aunque los hogares de nivel socioeconómico bajo se vean expuestos a porcentajes reducidos de amenazas, su vulnerabilidad puede ser alta debido a la materialidad de sus viviendas y la infraestructura deficiente del asentamiento, por ejemplo.

Figura $N^{0} 13$

Relación entre el porcentaje de población D y cobertura vegetal en la comuna de Peñalolén

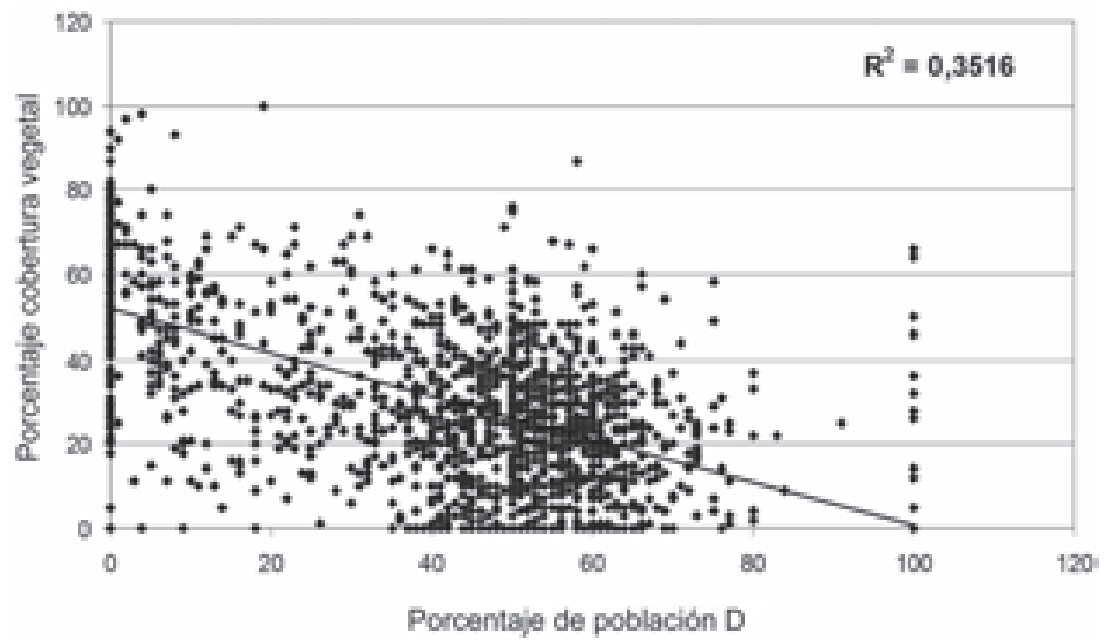

Fuente: Elaboración propia. 
En la Figura $N^{0} 14$ se observan las obras civiles como contenciones fluviales (Figura $\mathrm{N}^{\circ}$ 14-5) y canalizaciones (Figura $\mathrm{N}^{\circ}$ 14-1) destinadas a disminuir el peligro de inundación en dos quebradas del piedemonte andino de Peñalolén.

Sin embargo, es posible apreciar como estas obras de protección solo existen en los sectores dominados por GSE ABC1 y C2, y por el contrario, aguas abajo de las quebradas desaparecen, dejando mayormente expuestos a los grupos socioeconómicos más bajos.

\section{Consideraciones finales}

Los resultados muestran que existe una diferencia significativa en los riesgos ambientales a los que se ven expuestas las áreas habitadas por población de grupos socioeconómicos diferentes. En San Pedro de la Paz existe un mayor porcentaje de población pobre expuesta, perteneciente a los GSE D y E, debido a su localización temprana en zonas de gran amenaza de anegamiento, inundación, deflación eólica y mareas. Adicionalmente, son estos mismos espacios donde se localizan los verte-

Figura $N^{\circ} 14$

Distribución de obras civiles destinadas a disminuir la vulnerabilidad a inundaciones según niveles socioeconómicos de la población, en dos quebradas del piedemonte andino de Peñalolén

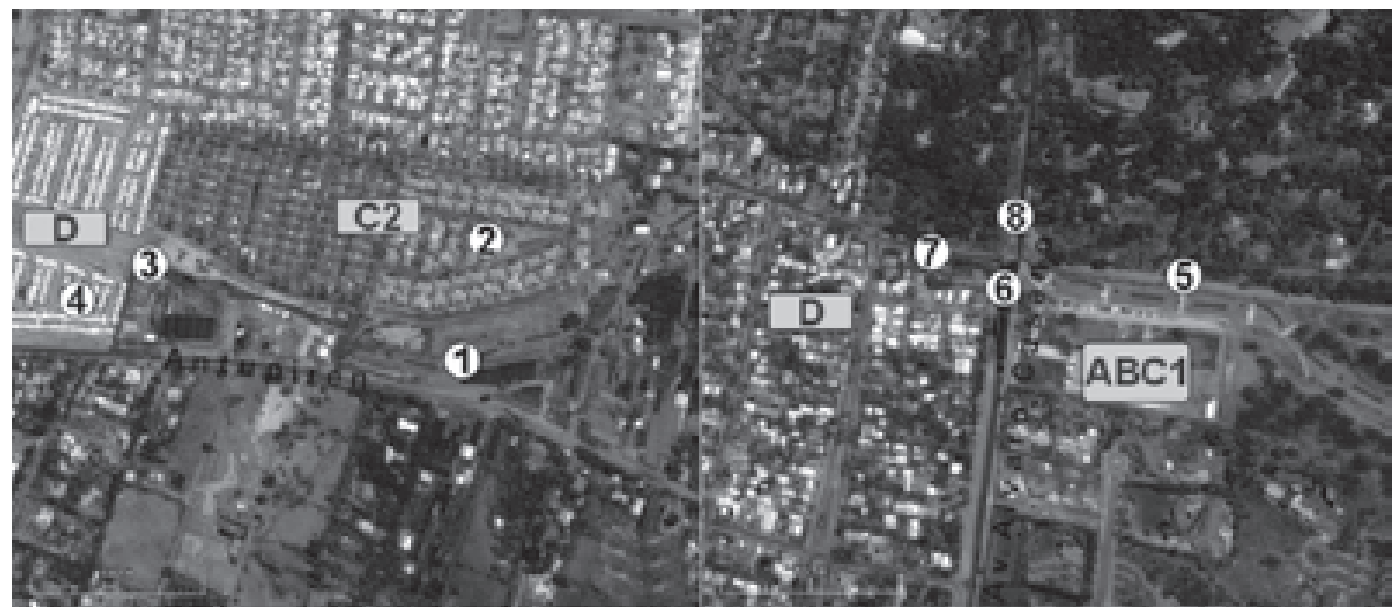

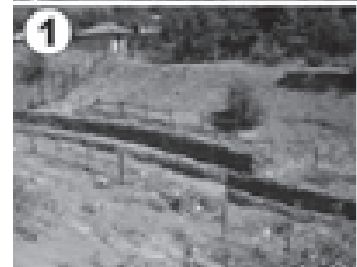
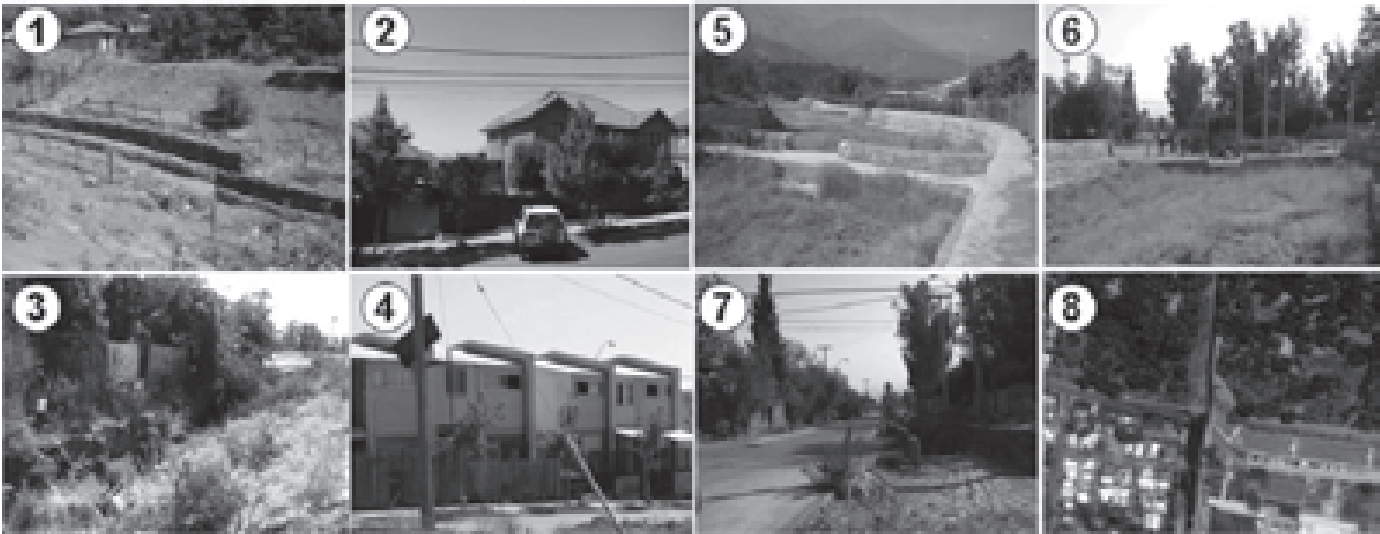

Fuente: Elaboración propia en base a Google Earth. 
deros ilegales, lo cual en su conjunto configura un escenario donde las amenazas son variadas y múltiples, causando impactos acumulativos y sinérgicos inexplorados aún.

En Peñalolén, la configuración del riesgo es similar a la descrita para San Pedro de la Paz, pero ha sido resultado de distintas combinaciones amenaza-vulnerabilidad, producto de que en Peñalolén el mayor porcentaje de población $A B C 1$ ha ocupado, mediante condominios cerrados, lugares del piedemonte andino de mayores amenazas pero, sin embargo, estos son los sectores menos vulnerables debido a su gran dotación de infraestructura y obras de mitigación para prevenir inundaciones, anegamientos y remociones en masa.

Esta situación se produce mediante el despliegue de los medios materiales y también intangibles por parte de los grupos $A B C 1$, que redunda en la disminución de su vulnerabilidad física frente a las amenazas analizadas $y$, por lo tanto, una menor exposición final a los riesgos.

\section{Referencias bibliográficas}

ADIMARK. Mapa socioeconómico de Chile, nivel socioeconómico de los hogares del país basado en datos del Censo. Investigaciones de mercado y opinión pública. Santiago: Adimark, 2002. Disponible en Internet: http://www.adimark.cl/medios/estudios/Mapa_Socioeconomico_de_Chile.pdf

BORSDORF, A.; HIDALGO, R. y SÁN$\mathrm{CHEZ}, \mathrm{R}$. Los mega diseños residenciales vaIlados en las periferias de las metrópolis latinoamericanas y el advenimiento de un nuevo concepto de ciudad. Alcances en base al caso de Santiago de Chile. En: CAPEL, H. e HIDALGO, R. (eds.). Construyendo la ciudad del siglo XXI. Retos y perspectivas urbanas en España y Chile. Santiago: Instituto de Geografía, Pontificia Universidad Católica de Chile, Serie GEOlibros Nº 6, 2006, p. 323-335.

CARDONA, O. La necesidad de repensar de manera holística los conceptos de vulnerabilidad y riesgo. Una crítica y una revisión necesaria para la gestión. Bogotá: Centro de
Estudios sobre Desastres y Riesgos (CEDERI), Universidad de los Andes, 2001. Disponible en Internet: http://www.desenredando.org/ public/articulos/index.html

DE LA MAZA, C.; HERNÁNDEZ, J.; BOWN, H.; RODRÍGUEZ, M. \& ESCOBEDO, F. Vegetation diversity in the Santiago de Chile urban ecosystem. Arboricultural Journal, 2002, No 26, p. 347-357.

ENVIRONMENTAL PROTECTION AGENCY (EPA). Guía del ciudadano para usar las leyes ambientales federales para asegurar justicia ambiental. Washington: EPA, 2002.

ESCOBEDO, F.; NOWAK, D.; WAGNER, J.; DE LA MAZA, C.; RODRÍGUEZ, M.; CRANE, D. \& HERNÁNDEZ, J. The socioeconomics and management of Santiago de Chile's public urban forests. Urban Forestry \& Urban Greening, 2006, № 4, p. 105-114.

FILGUEIRA, C. y PERI, A. América Latina: los rostros de la pobreza y sus causas determinantes. Santiago: CEPAL, Serie Población y Desarrollo No 54, 2004.

FISHER, J.; KELLY, M. \& ROMM, J. Scales of environmental justice: Combining GIS and spatial analysis for air toxics in West Oakland, California. Health \& Place, 2006, No 12, p. 701-714.

HIDALGO, R. De los pequeños condominios a la ciudad vallada: las urbanizaciones cerradas y la nueva geografía social en Santiago de Chile (1990-2000). Eure, 2004, vol. XXX, No 91, p. 29-52.

HIPPLE, J. Assesement of risk in urban environments using geo-spatial analysis. In: JENSEN, R.; GATRELL, J. \& MCLEAN, D. Geo-Spatial technologies in urban environments, policy, practice and pixels. Berlin: Springer-Verlag, 2007, p. 33-45.

INSTITUTO NACIONAL DE ESTADÍSTICAS (INE). XVII Censo de Población y VI de Vivienda. Santiago: INE, 2002.

IVERSON, L. R. \& COOK, E. A. Urban forest cover of the Chicago region and its relation to household density and income. Urban Ecosystems, 2000, № 4, p. 105-124. 
KRIEG, J. \& FABER, D. Not so black and white: environmental justice and cumulative impact assessments. Environmental impact assessment review, 2004, $N^{\circ} 24$, p. 667-694.

LAVELL, A. Desastres en América Latina: avances teóricos y prácticos: 1990-1999. En: Anuario Social y Político de América Latina y el Caribe. Caracas: FLACSO-Nueva Sociedad, 1999.

PEDLOWSKI, M.; CORABI, J. \& HEYNEN, N. Urban forest and environmental inequality in Campos dos Goytacazes, Rio de Janeiro, Brazil. Urban Ecosystems, 2002, № 6, p. 9-20.

ROMERO, H.; TOLEDO, X.; ÓRDENES F. y VÁSQUEZ, A. Ecología urbana y gestión sustentable de las ciudades intermedias chilenas. CIPMA. Revista Ambiente y DesarroIlo, 2001, vol. XVII, No 4, p. 45-51.

ROMERO, H.; VÁSQUEZ, A. y ÓRDENES, F. Ordenamiento territorial y desarrollo sustentable a escala regional, ciudad de Santiago y ciudades intermedias en Chile. En: FIGUEROA, E. y SIMONETTI, J. Globalización y biodiversidad: oportunidades y desafíos para la sociedad chilena. Santiago: Vicerrectoría de Investigación y Desarrollo de la Universidad de Chile, Editorial Universitaria, 2003, p. 167-224.

ROMERO, H. y VÁSQUEZ, A. Evaluación ambiental del proceso de urbanización de las cuencas del piedemonte andino de Santiago de Chile. Eure, 2005a, vol. XXXI, No 94, p. 97-117.
ROMERO, H. y VÁSQUEZ, A. La comodificación de los espacios urbanizables y la degradación ambiental en Chile. Scripta Nova, Revista Electrónica de Geografía y Ciencias Sociales, 2005b, vol. IX, Nº 194.

ROMERO, H. \& VÁSQUEZ, A. Geography for urban sustainable development: student's proposals to deal with Santiago de Chile urban sprawl. In: ROBERTSON, M. Sustainable Futures. Camberwell: ACER Press, 2007a, p. 125-151.

ROMERO, H. \& VÁSQUEZ, A. The Chilean free market and the lack of governance of urban green areas. In: HARTMUT, K. \& UWE T. (eds.). Berlin: $2^{\text {nd }}$ International Congress on Environmental Planning and Management. Visions, Implementations, Results. Landschaftsentwicklung und Umweltforschung, Schriftenreihe der Fakultät Planen Bauen Umwelt Band S20, 2007b, p. 267-269.

VARGAS, J. Políticas públicas para la reducción de la vulnerabilidad frente a los desastres naturales y socionaturales. Santiago: CEPAL, Serie Medio Ambiente y Desarrollo N $50,2002$.

VÁSQUEZ, A.; SALGADO, M.; ROMERO, A. \& SMITH, P. Socio-economic inequalities and the inequitable distribution of environmental risks in chilean metropolis local spaces: a perspective of environmental justice. São Paulo: Actas of 3rd International Seminar on Environmental Planning and Management, URBENVIRON São Paulo, 2008.

WALKER, G. \& BULKELEY, H. Geographies of environmental justice. Geoforum, 2006, No 37, p. 655-659. 